\title{
Modeling studies of potato nucleoside triphosphate diphosphohydro- lase NTPDase1: an insight into the catalytic mechanism
}

\author{
Anna Kozakiewicz ${ }^{1}$, Piotr Neumann ${ }^{1}$, Mariusz Banach², Michał Komoszyński² \\ and Andrzej Wojtczak ${ }^{1 凶}$ \\ ${ }^{1}$ Department of Crystallochemistry and Biocrystallography, Institute of Chemistry, Torun, Poland; ${ }^{2}$ Department \\ of Biochemistry, Institute of Biology, N. Copernicus University, Toruń, Poland
}

Received: 13 November, 2007; revised: 21 January, 2008; accepted: 05 March, 2008

available on-line: 11 March, 2008

\begin{abstract}
Nucleoside triphosphate diphosphohydrolase - NTPDase1 (apyrase, EC 3.6.1.5) was modeled based on sequence homology. The single polypeptide chain of apyrase is folded into two domains. The putative catalytic site with the apyrase conserved regions (ACR 1-5) is located between these two domains. Modeling confirmed that apyrase belongs to the actin superfamily of proteins. The amino acids interacting with the nucleoside triphosphate substrate and probably involved in the catalyzed hydrolysis were identified. The proposed two-step catalytic mechanism of hydrolysis involves Thr127 and Thr55 as potential nucleophilic factors responsible for the cleavage of the $P \gamma$ and $P \beta$ anhydride bonds, respectively. Their action seems to be assisted by Glu170 and Glu78 residues, respectively. The presence of two nucleophiles in the active site of apyrase explains the differences in the hydrolytic activity between apyrases and other enzymes belonging to the NTPDase family.
\end{abstract}

Keywords: NTPDase1, potato apyrase, homology modeling, enzymatic mechanism

\section{INTRODUCTION}

At least three groups of enzymes regulate the levels of cellular and extracellular nucleotides and nucleosides: nucleoside triphosphate diphosphohydrolases (NTPDases), pyrophosphatases/phosphodiesterases (NPPases), and alkaline phosphatases. The first group hydrolyzes only compounds with pyrophosphate bonds. Enzymes belonging to the two latter groups hydrolyze anhydride or ester bonds of nucleotides. Therefore, depending on the group of enzymes, the products of the catalyzed reaction might be either orthophosphate or pyrophosphate ions and the corresponding nucleoside monophosphate (NMP), nucleoside diphosphate (NDP) or nucleoside (Robson et al., 2006). High diversity of enzymes degrading nucleotides indicates the physi- ological importance of mechanisms controling nucleotide concentration. All NTPDases belong to the sub-class of ATP diphosphohydrolases (EC 3.6.1). These enzymes might be either soluble (Oses et al., 2004) or bound to the membranes. The catalytic site of enzymes occurring in the plasma membranes or in membranes of subcellular structures is located at the extracytoplasmic side of the membranes (Hanada \& Guidotti, 1996; Robson et al., 2006). In literature apyrases are classified as NTPDases1 based on their similar velocity of the ATP and ADP hydrolysis.

NTPDases are a structurally and catalytically homogeneous group of enzymes. Genes encoding these proteins have been found in vertebrates, invertebrates, plants and protozoans. The sequence identity among mammalian NTPDases varies between 24 and 96\%, while the identity between apy-

\footnotetext{
${ }^{\square}$ Corresponding author: Andrzej Wojtczak, Department of Crystallochemistry and Biocrystallography, Institute of Chemistry, N. Copernicus University, Gagarina 7, 87-100 Toruń, Poland; fax: (48) 56654 2477; e-mail: awojt@chem.uni.torun.pl Abbreviations: ACR, apyrase conserved region; ATPase, adenosine triphosphate hydrolase; ENTPDase, ecto-nucleoside triphosphate diphosphohydrolase; NDP, nucleoside diphosphate; NMP, nucleoside monophosphate; NTP, nucleoside triphosphate; NTPDase, nucleoside triphosphate diphosphohydrolase; NPPase, pyrophosphatase/phosphodiesterase; PDB, Protein Data Base; $\mathrm{P}_{\mathrm{i}^{\prime}}$ inorganic phosphate; PPX/GPPA, exopolyphosphatase/guanosine pentaphosphate phosphohydrolase; rms, root mean square; SA MD, simulated annealing molecular dynamics.
} 
rases (ATP diphosphohydrolase - EC 3.6.1.5) from pea or potato and mammalian NTPDases is only 23-24\% (Lavoie et al., 2004). These two groups of enzymes seem to have evolved from a common ancestor, but have separated at an early stage of evolution (Lavoie et al., 2004). Five characteristic apyrase conserved regions (ACR) are found in the sequences of NTPDases (Smith \& Kirley, 1999). Mutagenesis data show that these motifs are involved in substrate recognition, binding and hydrolysis (Kirley et al., 2006). NTPDases reveal a low substrate specificity, hydrolyzing purine and pyrimidine nucleoside di- and triphosphates. This might be explained by their high similarity in the ACR regions. However, different NTPDases hydrolyze nucleoside di- and triphosphates with different velocity (Kukulski \& Komoszyński, 2003; Kukulski et al., 2005). No consistent relations has been found between the residue mutations in ACR and the NTP or NDP preferences in the NTPDase family of enzymes (Robson et al., 2006).

The catalytic mechanism of ATP diphosphohydrolases is unclear. These enzymes, like potato apyrase (NTPDase1), hydrolyze ATP and ADP with similar velocities. The $K_{s h}$ value, which is the ratio of ATP to ADP hydrolysis velocities, is between 1 and 0.5 (Komoszyński \& Wojtczak, 1996; Kukulski \& Komoszyński, 2003; Kukulski et al., 2005). There are two general models of ATP hydrolysis. In the first model, ATP and ADP are hydrolyzed by the same domain of apyrase. The second model is based on the assumption that ADP produced in one active site is redirected to the other site and then hydrolyzed to AMP. The first model is supported by results of kinetic research on these enzymes. Hydrolysis of ATP or ADP by ENTPDase1 results in AMP and 1 or $2 \mathrm{P}_{\mathrm{i}}$ ions even after very short time. The $K_{\mathrm{M}}$ for ATP and ADP are equal (Kukulski \& Komoszyński, 2003). The second model was excluded by results of hydrolysis of an ATP-ADP mixture with the starting ATP concentration approximately $10^{3}$ times higher than that of ADP (Kukulski \& Komoszyński, 2003), still leading to the immediate production of AMP. Such a mechanism would require a very high enzyme affinity for ADP and a low affinity for ATP, in contradiction to the determined $K_{\mathrm{M}}$ values. Also, the high catalytic activity of apyrase (Hanada \& Guidotti, 1996) and the non-additive hydrolysis of ATP and ADP in equimolar concentrations (Kukulski et al., 2005) oppose such a mechanism. So far there is no report on the catalytic mechanism of apyrase at the molecular level.

Determination of the tertiary structure of NTPDases might give an insight into the catalytic mechanism of these enzymes. It was reported that these nucleotidases belong to the actin/Hsp70/sugar kinase superfamily of proteins (Smith \& Kirley,
1999). Therefore, the enzymes belonging to that superfamily might be structural templates for building an apyrase model. Lately, a theoretical model of human NTPDase3 was obtained based on the exopolyphosphatase 1T6C template (Ivanenkov et al., 2005). The aim of the reported research was to construct a 3-dimensional model of apyrase based on the sequence homology to members of the actin superfamily, and to propose a reaction mechanism based on the location of residues potentially involved in catalysis.

\section{METHODS}

The potato apyrase sequence derived from the corresponding cDNA sequence (Hanada \& Guidotti, 1996) was used for similarity analysis carried out via the Fold Recognition Meta-Server http:// www.genesilico.pl/meta (Kurowski \& Bujnicki, 2003), which uses freely available services for protein secondary structure prediction and fold recognition, among them MGENTHREADER (McGuffin \& Jones, 2003) and PSI-PRED (Jones, 1999). Only one sequence - PPX/GPPA exopolyphosphatase (Kristensen et al., 2004) - was chosen as the principal modeling template due to the highest score given consistently by all fold recognition services and significant score values allowing homology prediction. In particular, the similarity scoring function calculated with MGENTHREADER was 0.958 and indicated a reliable sequence alignment. Kristensen et al. (2004) described two structures of that exopolyphosphatase in an open and closed conformation (PDB codes: 1T6C and 1T6D, respectively). The rms difference between those structures is $0.64 \AA$, as calculated for $303 \mathrm{C} \alpha$ positions. Modeling of the 3dimensional structure of NTPDase1 was performed based on both these structures. The PPX/GPPA exopolyphosphatase also belongs to the actin superfamily (Kristensen et al., 2004). The sequence alignments produced by other servers (FFAS, FUGUE) (Rychlewski et al., 2000; Shi et al., 2001) were similar to that obtained with MGENTHREADER.

Potato apyrase is larger than PPX/GPPA exopolyphosphatase due to the presence of some long insertions (Met1-Glu42, Glu224-Arg296 regions) that are absent in the template structure. The Met1-Glu42 region was modeled using the Arg8-Lys47 N-terminal fragment of ribosomal protein S15 (Clemons et al., 1998) (PDB code: 1A32). This protein was chosen based on similarity of the secondary structure and subsequent sequence-sequence alignment with T-coffee (Notredame et al., 2000). A large insertion (Glu224-Arg296) occurs in the position corresponding to sub-domain 4 of exopolyphosphatase (Fig. 1). An analogous domain with a similar length but low 


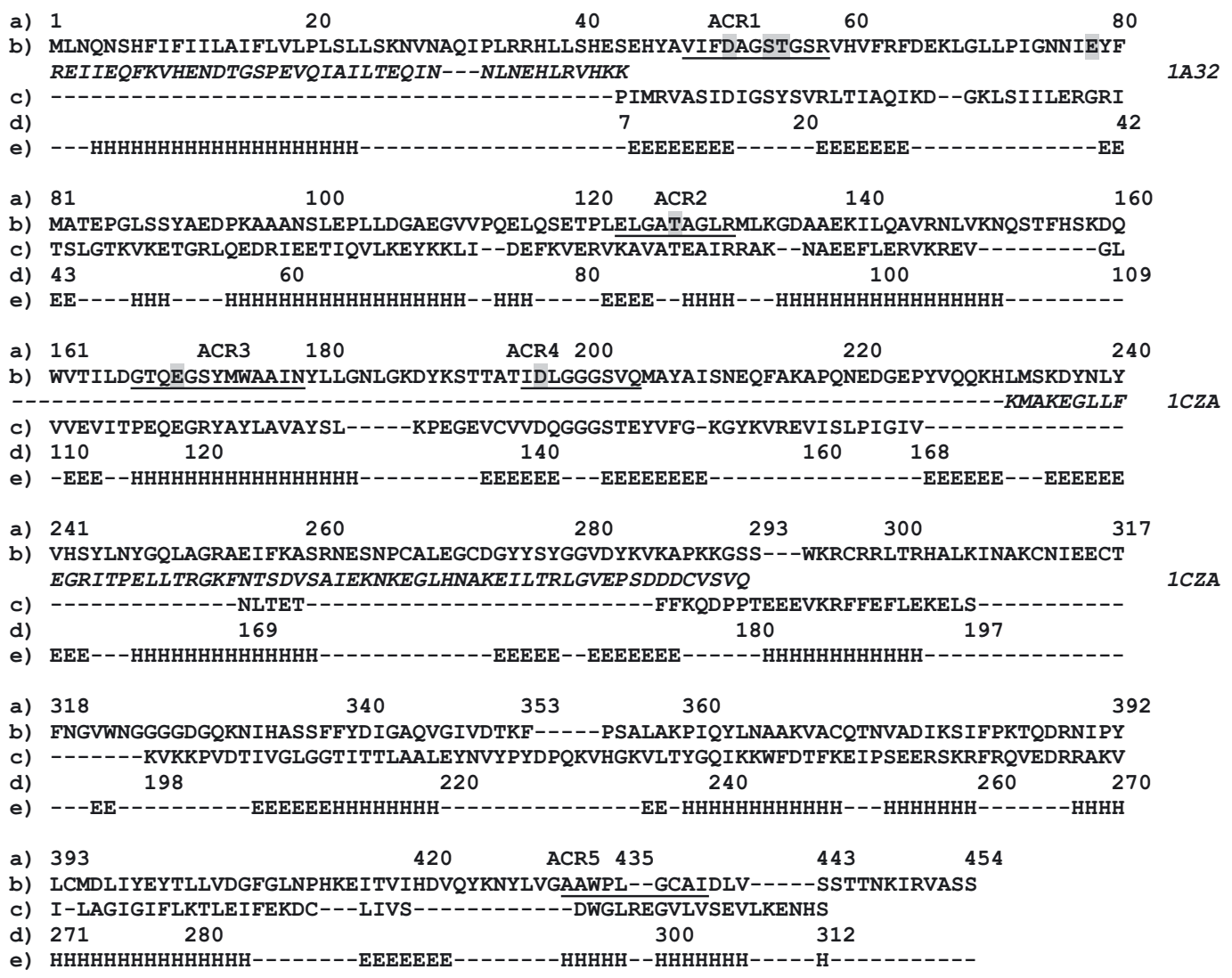

Figure 1. Final sequence alignment of potato apyrase (b) with exopolyphosphatase 1T6D (c) as produced by MGENTHREADER, the residue and ACR numbering for apyrase (a) and the residue numbering of exopolyphosphatase (d), the secondary structure of apyrase as predicted by PSI-PRED (e).

The ACR regions in apyrase are underlined, while residues indicated by modeling as important for the catalytic activity of apyrase are shaded. Additional lines (in italics) below the sequence of apyrase indicate the sequence fragment for S15 protein fragment (PDB code 1A32, residues 8-47) for the N-terminal stretch and the hexokinase domain (1CZA, residues 312-372) similar in fold to domain 4 of NTPDase1 with their PDB code. Their alignment was made based on the predicted secondary structure, since the sequence similarity to that of NTPDase1 is low.

sequence similarity was found in other proteins of the actin superfamily. Among those, hexokinase 1CZA (Aleshin et al., 2000) revealed both ends of the domain positioned similarly to those expected for the apyrase model based on the 1T6C/1T6D structure, with the secondary structure resembling that predicted for apyrase with PSI-PRED. Therefore the 312-372 fragment of hexokinase 1CZA was aligned with the T-coffee program and selected as a template for modeling of that apyrase stretch. The constructed apyrase molecule was modeled with the MODELLER program (Fiser et al., 2000). A hybrid model was created from fragments of preliminary models taking into account the score for each amino acid calculated by the PROSA program (Sippl, 1993). That hybrid model was superimposed onto the template (1T6C/1T6D structure). Based on this superposition, a target-template sequence alignment was inferred and used for building a new model with MODELLER. Each time the template structure was included in the modeling procedure. The qual- ity of the new interim models was re-evaluated using PROSA and PROCHECK (Laskowski et al., 1993) programs and the alignments were improved by shifting regions with unsatisfactory PROSA scores. The regions with positive PROSA scores belonged mostly to the two long insertions mentioned above and some loops on the protein surface. During the modeling procedure, the five ACR regions were strictly conserved. The improved alignments were used for building new generation models. Only one region at a time was shifted to avoid the interference of effects from different parts. At this stage additional informations about secondary structure, placement of insertions and deletions in loops, as well as conservation of residues (five ACR regions) of the reference exopolyphosphatase were taken into account to verify the robustness of the model. The improved models were again evaluated using PROSA and PROCHECK programs and the whole procedure was repeated until all regions had a negative PROSA score, reflecting preferable interactions with 
the surrounding residues and good stereochemistry. The model obtained with 1T6D had a better PROSA score and conformation of the polypeptide chain as determined with PROCHECK. Verification of stereochemistry of the apyrase model based on 1T6D with PROCHECK gave $88.9 \%$ of residues in the most favorable regions of the Ramachandran plot, while the remaining $11.1 \%$ residues were in the allowed regions. The corresponding statistics for the model based on 1T6C was $85.9 / 14.1 \%$. Therefore, the reported model of NTPDase was based on 1T6D as the primary template (Kristensen et al., 2004). The final sequence superposition used for constructing the model is shown in Fig. 1. The manual shifts relative to the MGENTHREADER results are reflected in the alignment as gaps occurring at positions 293, 353, 435 and 442. Superposition of the reported apyrase model with the structures of proteins belonging to the actin superfamily allowed the position of ATP and the metal ion to be identified. The ATP ligand and the $\mathrm{Ca}^{2+}$ ion were built into the model based on a superposition with actin-ATP complexes (Kabsch et al., 1990). The model of the apyrase-ATP-Ca ${ }^{2+}$ ternary complex was then subjected to simulated annealing molecular dynamics (SA MD) minimization using the CHARMM force field as implemented in the CNS program package (Brünger et al., 1998). Finally, water molecules were added with the VMD program (Humphrey et al., 1996) and the model was minimized with the NAMD program, using the CHARMM27 force field (Phillips et al., 2005). Figures of the molecule were prepared with MOLSCRIPT (Kraulis, 1991) and RASTER3D (Merritt \& Bacon, 1997).

\section{RESULTS AND DISCUSSION}

The PROSA (Sippl, 1993) score was negative for most of the final model of potato apyrase, which

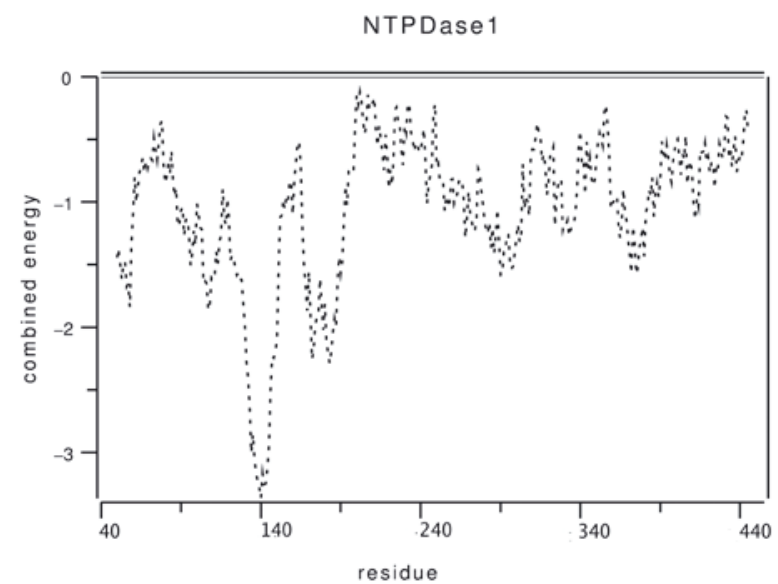

Figure 2. PROSA score for the final model of potato apyrase with residues 1-40 omitted. indicates its correctness (Fig. 2). The only section of the initial model with a positive score corresponded to the highly hydrophobic fragment at the N-terminus (residues 1-40). That $\mathrm{N}$-terminal fragment was identified as an apparent trans-membrane domain using PFAM (Bateman et al., 2004) and included into initial modeling. However, Handa and Guidotti (1996) interpreted that fragment as a signal peptide. Therefore we excluded it from the final model of NTPDase 1. The rms difference between the template 1T6D structure and the reported model of potato apyrase, shown in Fig. 3, is $1.22 \AA$ calculated for 268 positions of $\mathrm{C} \alpha$ atoms.

\section{The overall structure of apyrase molecule}

The structure of potato apyrase is similar to structures of proteins belonging to the actin superfamily (Hanada \& Guidotti, 1996; Smith \& Kirley, 1999). Therefore, the domain numbering in Fig. 3 is analogous to that used in actin (Schüler, 2001). The molecule is built of four sub-domains assembled to form two domains of the ribonuclease $\mathrm{H}$ architecture (mixed five-stranded $\beta$-sheet and four or five $\alpha$ helices; PROSITE documentation entry PDOC50879) (Yang et al., 1990). These domains are separated by the central cleft which contains the active site in actin and related proteins. Similar to the template exopolyphosphatase, the apyrase molecule has a central helix (residues 167-185) which corresponds to the ACR3 region. In exopolyphosphatase, that re-

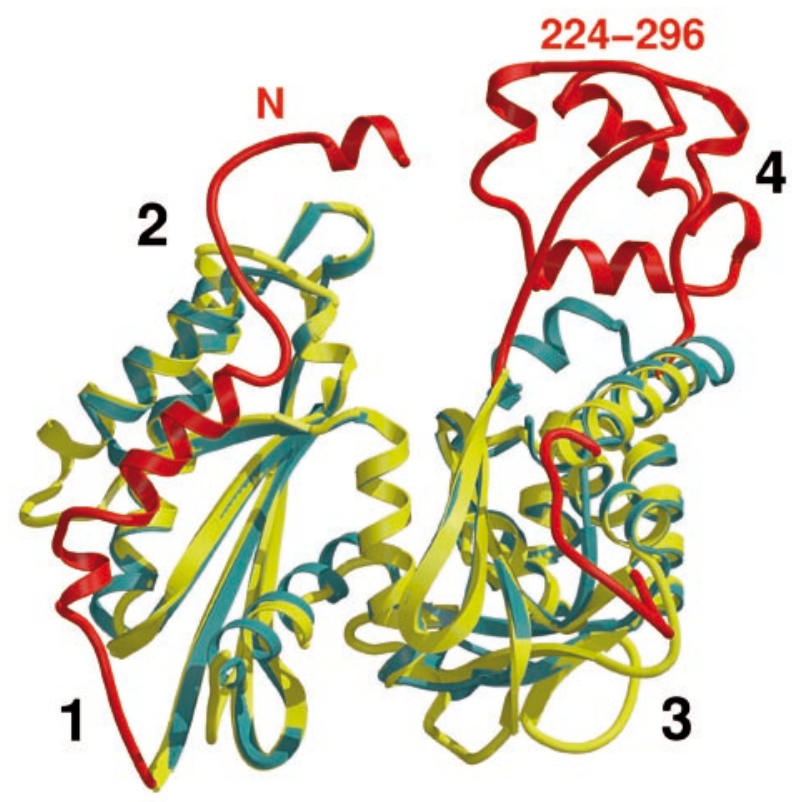

Figure 3. Superposition of the apyrase model (yellow) and exopolyphosphatase 1T6C (green).

Fragments of the apyrase molecule having no equivalents in the $1 \mathrm{~T} 6 \mathrm{C}$ template structure are colored red. The subdomain numbering is analogous to that used for actin (Schüler, 2001). 
gion was found responsible for the re-orientation of subdomains relative to each other (1T6C vs. 1T6D) in the open and closed conformations of the enzyme (Kristensen et al., 2004), changes interpreted as part of the enzymatic mechanism (Kirley et al., 2006).

The major difference between the reported apyrase model and exopolyphosphatase is sub-domain 4 . In 1T6D, that sub-domain consists of very few amino acids (residues 167-174). In apyrase it comprises a large fragment (residues 224-296), with only low sequence similarity to other proteins of the actin superfamily. The function of sub-domain 4 in apyrase is unknown. In actin, the corresponding domain 4 and domain 2 are involved in formation of a complex with DNase1 (Kabsch et al., 1990), while in Hsp70 they interact with the BAG co-chaperone (Sondermann et al., 2001). Ecto-apyrases are transmembrane proteins and some of them reveal sequence similarity to cell adhesion molecules (Auryvilius et al., 1990; Komoszyński \& Wojtczak, 1996). Therefore, one might hypothesize that these domains of apyrase, positioned on the opposite side of the molecule than the potential trans-membrane $\mathrm{N}$ terminal fragment, might be involved in interactions with other proteins.

The tertiary and quaternary structure of extracellular proteins is frequently stabilized by disulfide bridges. The theoretical model of human NTPDase3

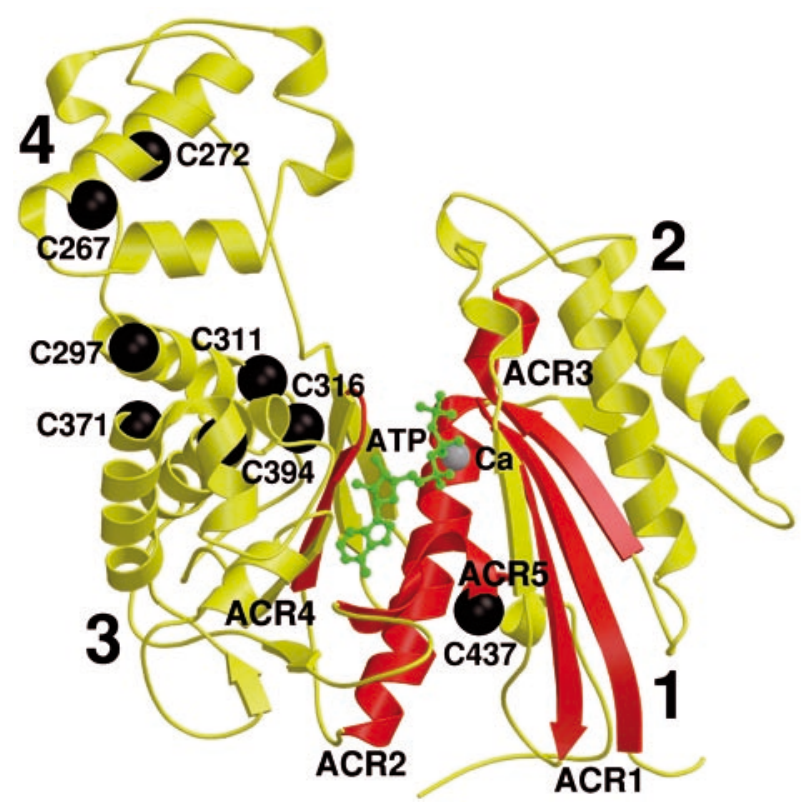

Figure 4. Apyrase model containing residues 40-454, with ACR regions (red).

The central cleft contains the putative catalytic site with ATP and $\mathrm{Ca}^{2+}$ displayed in positions achieved after the CHARMM force field optimization with the simulated annealing molecular dynamics protocol. Cysteine residue positions are indicated by black spheres. The $\mathrm{N}$ - and $\mathrm{C}$ termini are also labeled. The sub-domain numbering is analogous to that used for actin (Schüler, 2001). and mutagenesis data indicated the presence of five disulfide bridges, crucial for the enzymatic activity (Ivanenkov et al., 2005). The architecture of the apyrase model reported here and that of NTPDase3 are almost identical. The apyrase model was built with no explicit constraints for the disulfide bridge formation. Out of the eight Cys residues present in potato apyrase, three cannot form any disulfide bridge due to their position in the molecule (Cys267, Cys272 and Cys437). The pair of Cys311 and Cys316 is located within the same $\alpha$-helix, but being separated by four other residues, they could barely form a disulfide bridge (Fig. 4). The other three Cys residues (Cys297, Cys371 and Cys394) are located near each other and two possible bridges Cys371-Cys297 or Cys371-Cys394 could be formed. However, all Cys residues in apyrase are distant from the putative catalytic site and the potential formation of disulfide bridges should not affect the overall architecture of the molecule. Therefore, the apyrase model indicates they would not affect significantly the catalytic activity of apyrase. That conclusion is consistent with the reported insensitivity of the enzymatic activity of plant apyrases to $\mathrm{SH}$ group reagents (Komoszyński, 1996).

Structural elements possibly related to catalytic activity

In general opinion, the ACR regions should coincide with the catalytic center due to their conservation in apyrases. The differences in the active site residues should explain the observed differences in the enzymatic activity. Therefore, we compared the model structure and the ACR location with those reported for proteins of known crystal structures actin, Hsp70 and PPX/GPPA exopolyphosphatase.

The ACR regions identified for the potato apyrase (Hanada \& Guidotti, 1996), as well as the regions expected to be involved in the apyrase activity and corresponding to the Serano motifs of ATPases (Komoszyński \& Wojtczak, 1996), constitute the putative active site in our model (Fig. 4). They correspond to the ACR location in the exopolyphosphatase 1T6D and NTPDase3 (Ivanenkov et al., 2005). Such an architecture suggests the mechanism of ATP and ADP hydrolysis in a single catalytic site.

The ACR1 fragment was reported to be responsible for phosphate binding (Kristensen et al., 2004) and possibly involved in hydrolysis of the respective pyrophosphate bond. In the apyrase model, the ACR1 and ACR2 fragments are located near the putative active site and are involved in the interactions with the metal ion and the substrate. The fragment corresponding to apyrase ACR3 is involved in the metal positioning interactions and is reported to be important for the hydrolytic process in actin 
and Hsp70 (Wilbanks et al., 1994; Sriram et al., 1997). The role of the conserved ACR4 region in enzymes belonging to the actin superfamily is to bind the $\beta$ phosphate. That ACR was reported to participate in metal binding and to contain the hydrolytically active residues in actin (Vorobiev et al., 2003) and human brain ecto-apyrase HB6 (Smith \& Kirley, 1999).

The apyrase C-terminal fragment contains the ACR5 region (residues Ala431-Ile439) and is part of the active site. The superposition reveals that the position of this fragment is different between the apyrase model and $1 \mathrm{~T} 6 \mathrm{D}$, but their position relative to other ACRs is the same in both structures. It is difficult, however, to make a reasonable interpretation of that shift since in $1 \mathrm{~T} 6 \mathrm{D}$ the C-terminal fragment is much shorter than in apyrase. In the apyrase model the helix with ACR5 is followed by a fragment in $\beta$ conformation, which enforces the shift of the helix. The conformational change is also due to the 12 amino-acid insertion prior to that helix. On the other hand, the position of Trp433 of ACR5 is identical to that of the corresponding Trp295 of 1T6D. That residue was reported to be an important part of the nucleotide-binding pattern (Smith et al., 1999) in NTPDase3.

\section{Metal site}

In the actin superfamily, the hydrolytic activity and the substrate specificity are regulated by the kind of the metal ion bound in the active site. The apyrase activity is also modulated by divalent metal cations. The major role is reported for $\mathrm{Ca}^{2+}$ and
$\mathrm{Mg}^{2+}$. It was found that calcium enhances the ADPase activity while magnesium enhances the ATPase activity of Solanum tuberosum apyrase, therefore affecting the $K_{\mathrm{sh}}$ ratio of that enzyme (Komoszyński \& Wojtczak, 1996). Binding of $\mathrm{Mg}^{2+}$ ions is reported to cause a 20-fold increase in the ATPase activity of actin compared to that in the presence of $\mathrm{Ca}^{2+}$ (Schüler et al., 1999; Schüler, 2001). That clearly indicates a similarity in the catalytic mechanism between actin and apyrase. Since the architecture of the active site in the actin and Hsp70 ATPase is similar, it seems probable that similar residues could be involved in the metal binding by apyrase (Wilbanks et al., 1994).

In the apyrase model reported here, the residues involved in the metal binding are Glu170, Asp51 and Asp197 (Fig. 5). Their distances to the metal ion in apyrase are between 4 and $7 \AA$, and those interactions are mediated by the coordinated water molecules. Both the structural reports and mutagenesis data showed that in the actin superfamily the metal ions are bound to the carboxylic groups of the conserved residues (Wilbanks et al., 1994; Smith \& Kirley, 1999; Vorobiev et al., 2003) (Table 1). In apyrase, the relative position of the metal ion and ATP is similar to those reported for the actin or Hsp70 structures. In all these structures the metal ion either directly coordinates the $\beta$ and $\gamma$ phosphates of ATP, or participates in the ATP positioning via a network of $\mathrm{H}$-bonds involving water molecules of the metal coordination sphere. Such metal position also maintains the conformation of the triphosphate moiety required for hydrolysis. Therefore, any substitution of the amino

Table 1. Amino-acid residues hypothetically involved in the catalytic action of NTPDase1 and their equivalents in enzymes belonging to the actin superfamily.

The apyrase residues are identified by a least-squares superposition of the corresponding crystal structures with the apyrase model reported in this paper.

Location of the residue

\begin{tabular}{|c|c|c|c|c|c|c|c|c|}
\hline \multirow[b]{2}{*}{ Apyrase } & \multicolumn{3}{|c|}{ ACR1 } & \multirow{2}{*}{$\begin{array}{c}\text { LOOP } 1 \\
\text { Glu78 }\end{array}$} & \multirow{2}{*}{$\begin{array}{l}\text { ACR2 } \\
\text { Thr127 }\end{array}$} & \multirow{2}{*}{$\begin{array}{c}\text { ACR3 } \\
\text { Glu170 }\end{array}$} & \multicolumn{2}{|c|}{ ACR4 } \\
\hline & Ser54 & Thr55 & Asp51 & & & & Asp197 & Gln204 \\
\hline $\begin{array}{l}\text { NTPDase3 * } \\
\text { (model } 5 \text { FFAS) }\end{array}$ & Ser65 & Ser66 & Asp62a, d & Cys92 & Thr139 & Glu182a, h & Asp219a & $\mathrm{Gln} 226^{\mathrm{a}, \mathrm{h}}$ \\
\hline $\begin{array}{l}\text { Actin } \\
\text { (PDB code 1atn) }\end{array}$ & Ser14 & Gly $15^{f}$ & Asp11 ${ }^{\mathrm{f}}$ & Pro32 & Gly74 & $\mathrm{Gln} 137^{\mathrm{f}}$ & Asp154 & His $161^{f}$ \\
\hline $\begin{array}{l}\text { HSP70 } \\
\text { (PDB code 1s3x) }\end{array}$ & Thr13e & Thr14 & Asp10g & Asp69 & Lys7 $^{\mathrm{b}}$ & Glu175g & Asp199g & Asp206g \\
\hline $\begin{array}{l}\text { PPX/GPPA Exopolyphosphatase } \\
\text { (PDB code } 1 \text { t } 6 \mathrm{~d} \text { ) }\end{array}$ & Ser18 & Y19 & Asp15 & Ala42 & Thr87 & Glu119 & Glu148 & Asp141 \\
\hline
\end{tabular}

*NTPDase3 model - from Ivanenkov et al. (2005) - model 5 'FFAS_NTPDase3_3D_coordinates_with_side_chains.pdb'. The role of the residues is described in the respective references. a) Kirley et al. (2006), b) O'Brien et al. (1996), c) Schüler et al. (1999), d) Smith \& Kirley (1999), e) Sousa \& MacKay (1998), f) Vorobiev et al. (2003), g) Wilbanks et al. (1994), h) Yang et al. (2001). 
acids contacting the metal ion in apyrase should affect the enzyme activity by changing the affinity of the metal site or by influencing the $\mathrm{Me}^{2+}$ position within the active site.

\section{Hypothetic mechanism of ATP hydrolysis by apy-} rase

The mechanism of the catalytic hydrolysis of ATP has been studied for different members of the actin superfamily, but there are no reports on the possible mechanism of the reaction catalyzed by apyrase at the molecular level. Analysis of literature reports suggests that there is no single universal mechanism for these enzymes. However, a comparison of different enzymes superimposed on the apyrase model enables some suggestions to be made for the possible molecular basis of the apyrase catalytic mechanism.

According to the mechanism proposed for actin, the cleavage of the $\gamma$ phosphate is due to the activation of the nucleophilic water or a hydroxyl ion by His161 (ACR4 region) and its positioning by Gln137 (Vorobiev et al., 2003). In the apyrase model, Gln204 and Glu170 corresponding to the actin residues (Table 1) are distant from the ATP position and could not be involved in direct interactions with the potential nucleophile required for the $\mathrm{P} \gamma$ cleavage or further conversion of ADP to AMP. Therefore, the mechanism of apyrase action seems to be different from that suggested for actin. On the other hand, Glu170 in our model is involved in interactions with the metal cation, and might be important for the apyrase activity.

Another possible mechanism of the apyrase action might be similar to that suggested for Hsp70 ATPase. That mechanism is based on the activation of the nucleophilic water by Lys71, which is H-bonded to Glu175 (Sriram et al., 1997; Sousa \& MacKay,

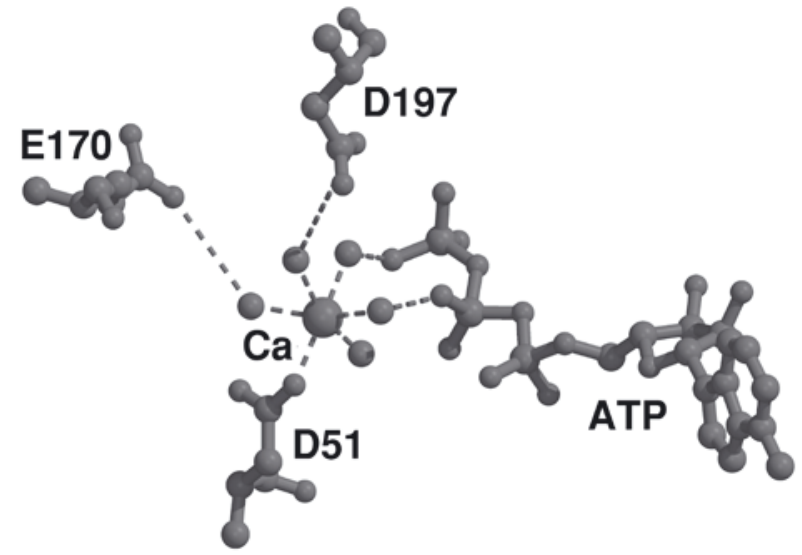

Figure 5. Metal binding site and ATP positioning interactions in potato apyrase.
1998), and its attack on $\gamma$ phosphate of ATP. The participation of both these residues in the mechanism is confirmed by mutagenesis data (Wilbanks et al., 1994; O'Brien et al., 1996). In apyrase, the corresponding residues are Thr127 and Glu170 (Table 1). At $\mathrm{pH}$ 6.5, optimal for apyrase, that threonine might play a role of the nucleophile activator, enhanced by the interaction with Glu170. However, their position is not appropriate for cleavage of the $\beta$ phosphate moiety. Since apyrase hydrolyzes both ATP and ADP to adenosine monophosphate and inorganic orthophosphate ions, that site cannot be the only one taking part in the reaction, unless the enzyme molecule undergoes a dramatic conformational re-arrangement between the first and second step of ATP hydrolysis.

The apyrase model suggests another possible mechanism of hydrolysis. Two polar residues of ACR1, Ser54 and Thr55, are positioned near $\gamma$ and $\beta$ phosphates of ATP, respectively. Both these residues might participate in the ATP conversion to AMP, either by activation of the catalytic water molecule or with the formation of phosphorylated intermediates. The cleavage performed by Ser54 would produce ADP, which in turn would be further hydrolyzed with Thr55 involved (Fig. 6).

The participation of Ser54/Thr55 in the catalysis would explain the dual activity of apyrase in a satisfactory way. There are experimental data indicating the importance of these residues for ATP hydrolysis. The S57A substitution of the Ser54 analogue in CD39 (Drosopoulous et al., 2000) is reported to increase the ADPase activity and decrease the ATPase activity of CD39 apyrase. The mechanism involving Ser54 would require the presence of additional residues assisting in the nucleophile activation, but our model does not reveal any such residue near its

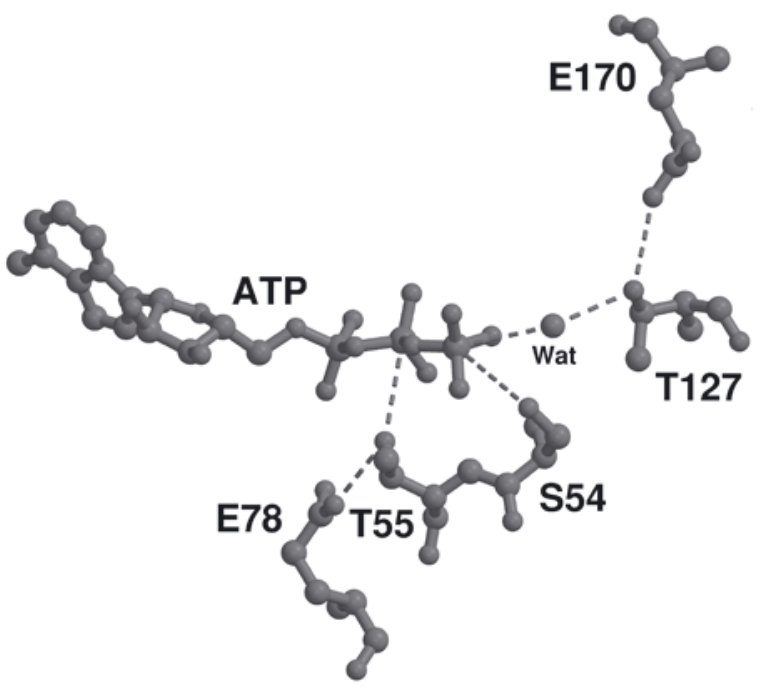

Figure 6. Catalytic site of apyrase with residues potentially involved in ATP hydrolysis to AMP. 
side chain. Formation of two phosphorylated species on Ser54 and Thr55, and subsequent release of two orthophosphates would explain the differences between the activity of apyrase and other members of the actin superfamily. There is no direct evidence of the formation of the phosphorylated intermediates during hydrolysis catalyzed by potato apyrase. However, a sequence comparison to the Serano motifs of ATPases suggested possible phosphorylation sites, including ones in the ACR1 conserved region (Komoszyński \& Wojtczak, 1996).

The mutagenesis data indicated that in actin and Hsp70 the residues equivalent to Ser54 are involved in the ATP binding rather than in the hydrolysis. In Hsp70, Thr13 and Thr14 are located almost identically to the apyrase residues 54 and 55 mentioned above. However, substitution of Thr13 did not change the hydrolytic activity of Hsp70 ATPase (Sousa \& MacKay, 1998). Therefore, in that enzyme the side chain of Thr13, located near the triphosphate moiety of ATP, seems to be involved only in the ATP positioning. In actin the pair Ser14/ Gly15 corresponds to the apyrase Ser54/Thr55. The S14A or S14C variants reveal normal hydrolytic activity (Chen \& Rubenstein, 1995; Schüler et al., 1999). Therefore, Ser14 does not play a role of the primary nucleophile and actin has only the ATPase activity. Based on the similarity of these enzymes, one might expect that the apyrase Ser54 would also be only responsible for positioning of ATP in the catalytic site.

The second residue, Thr55, is positioned between the $\beta$ - and $\gamma$-phosphate moieties of ATP and could participate in the cleavage of the $\mathrm{P} \beta$ bond. There is Glu78 which might cooperate with Thr55 in that hydrolysis (Fig. 6). Biochemical data do not reveal the presence of pyrophosphate among the products of enzymatic conversion of ATP by apyrase (Komoszyński \& Wojtczak, 1996). The orchestrated action of apyrase Ser54 and Thr55 would be consistent with the detected AMP and orthophosphate ions as the only products of the catalyzed reaction. An alternative explanation might be the participation of Thr55 in a two-step hydrolysis with a nucleophilic attack on both phosphates. If Thr55 is the only catalytic nucleophile, pyrophosphate should be detected, unless there is a strong preference to cleave the $\gamma$ phosphate first. However, such a single-nucleophile mechanism with Thr55 should be excluded, since potato apyrase hydrolyzes ATP and ADP with similar velocity (Komoszyński \& Wojtczak, 1996; Kukulski \& Komoszyński, 2003). Consequently, there should be a two step mechanism of ATP to AMP hydrolysis by apyrase. In Hsp70, Thr14 occupies the position equivalent to apyrase Thr55 and might play a similar role. The residual ADPase activity reported for Hsp70 (Mayer \& Bukau, 2005) seems to be consistent with the hypothetical involvement of apyrase
Thr55 in the hydrolysis. On the other hand, in actin the corresponding Gly15 cannot participate in the hypothetical hydrolysis of $\beta$ phosphate.

Superposition of the NTPDase3 (Ivanenkov et al., 2005) and apyrase models explains the reported differences in the substrate specificity of these enzymes. Apyrase (NTPDase1) hydrolyzes ATP and ADP equally efficiently, while NTPDase3 reveals an approximately 3-fold preference for ATP over ADP. In both molecules, the regions suggested by the apyrase model as being responsible for the $\mathrm{P} \gamma$ cleavage are identical, with Ser54, Thr127 and Glu170 of NTPDase1 corresponding to Ser65, Thr139 and Glu182 in the NTPDase3 model, respectively. However, the residues responsible for the $\beta$-phosphate hydrolysis in apyrase are Thr55 and Glu78, while in NTPDase3 their structural equivalents are Ser66 and Cys92, respectively, and there is no functional equivalent of apyrase Glu78 near the position of Ser66 in NTPDase3. This seems to explain the less efficient hydrolysis of ADP by NTPDase3, when compared to apyrase. That conclusion is consistent with the mutagenesis data summarized by Kirley et al. (2006) and further supports the theoretical model of apyrase reported here and the proposed mechanism of apyrase action.

The NTPDase1 model reported here suggests a mixed enzymatic mechanism consisting of elements analogous to those suggested for Hsp70, and the Thr55 involvement described above. In the first step the $\mathrm{P} \gamma$ cleavage would be performed by the Thr127...Glu170 pair, while the subsequent hydrolysis of the $\beta$-phosphate would be performed by the Thr55...Glu78 pair. In our model, all these residues are positioned appropriately for conducting such reactions (Fig. 6). Although the detailed mechanism of the ATPase activity of apyrase (Thr127 or Ser54) is unclear, the reported model gives the structural basis for explanation of its ADPase activity. The two-step mechanism derived from the apyrase model indicates different sets of residues involved in the cleavage of $\gamma$ - and $\beta$-phosphate moieties. Such a model is consistent with the experimental data on both mutagenesis and the products of the ATP conversion. The mechanism proposed here also explains the differences in the catalytic activity between members of the actin superfamily.

\section{CONCLUSIONS}

The consistency of the homology modeling results with the available activity data confirm that potato apyrase belongs to the actin superfamily. The molecule consists of two domains having the ribonuclease $\mathrm{H}$ architecture. The ACR position suggests that the central cleft of the molecule contains the 
active site of the enzyme. The structure of the catalytic cleft shows the position of ATP and the metal ion binding interactions, both consistent with the biochemical data on apyrases. The reported model is consistent with the available experimental data on the activity of actin, Hsp70, enzymes from the NTPDase group, and their variants. The model explains the differences in the catalytic activity between apyrases and ATPases and reveals residues that could participate in the hydrolysis of ATP to AMP. The most probable mechanism of $\mathrm{P} \gamma$ cleavage would involve a water molecule activated by the Thr127... Glu170 pair of residues (equivalent to Hsp70 Lys71... Glu175). Another pair Thr55...Glu78 would participate in the $P \beta$ cleavage. The reported model supports the hydrolytic mechanism based on a single active site. Another interesting option would be a mechanism based on the nucleophilic attack assisted by Ser54 and subsequently by Thr55, which would not require any major conformational adjustments in the active site and would be consistent with the reported lack of pyrophosphate among the reaction products. The model points out to Glu170, Asp51 and Asp197 as residues involved in positioning the metal ion required for the catalytic activity of apyrase. The hypothesis on the involvement of all the mentioned residues in the catalysis, derived from the position similarity to the active residues of other enzymes, is consistent with the available mutagenesis data.

\section{Acknowledgements}

Research was partly supported by grant Ch-511 of the Rector of the Nicolaus Copernicus University, Toruń, Poland.

\section{REFERENCES}

Aleshin AE, Kirby Ch, Liu X, Bourenkov GP, Bartunik HD, Fromm HJ, Honzatko RB (2000) Crystal structures of mutant monomeric hexokinase I reveal multiple ADP binding sites and conformational changes relevant to allosteric regulation. J Mol Biol 296: 1001-1015.

Auryvilius M, Hansen OC, Lazarek MBS, Bock E, Obrink B (1990) The cell adhesion molecule Cell-CAM 105 is an ecto-ATPase and member of the immunoglobulin superfamily. FEBS Lett 264: 267-269.

Bateman A, Coin L, Durbin R, Finn RD, Hollich V, Griffiths-Jones S, Khanna A, Marshall M, Moxon S, Sonnhammer ELL, Studholme DJ, Yeats C, Eddy SR (2004) The Pfam protein families database. Nucleic Acids Res 32: D138-D141.

Brünger AT, Adams PD, Clore GM, DeLano WL, Gros P, Grosse-Kunstleve RW, Jiang JS, Kuszewski J, Nilges M, Pannu NS, Read RJ, Rice LM, Simonson T, Warren GL (1998) Crystallography and NMR system: a new software suit for macromolecular structure determination. Acta Crystallogr D Biol Crystallogr D54: 905-921.
Chen X, Rubenstein PA (1995) A mutation in an ATP-binding loop of Saccharomyces cerevisiae actin (S14A) causes a temperature-sensitive phenotype in vivo and in vitro. J Biol Chem 270: 11406-11414.

Clemons JWM, Davies C, White SW, Ramakrishnan V (1998) Conformational variability of the N-terminal helix in the structure of ribosomal protein S15. Structure 6: $429-438$.

Drosopoulous JHF, Broekman MJ, Islam N, Maliszewski ChR, Gayle RB III, Marcus AJ (2000) Site-directed mutagenesis of human endothelial cell ecto-ADPase/Soluble CD39: Requirement of glutamate 174 and serine 218 for enzyme activity and inhibition of platelet recruitment. Biochemistry 39: 6936-6943.

Fiser A, Do RK, Sali A (2000) Modeling of loops in protein structures. Protein Sci 9: 1753-1773.

Hanada M, Guidotti G (1996) Purification and cloning of a soluble ATP-diphospohydrolase (apyrase) from potato tubers (Solanum tuberosum). Biochem Biophys Res Commun 218: 916-923.

Humphrey W, Dalke A, Schulten K (1996) VMD - visual molecular dynamics. J Mol Graphics 14: 33-38.

Ivanenkov VV, Meller J, Kirley TL (2005) Characterization of disulfide bonds in human nucleoside triphosphate diphosphohydrolase 3 (NTPDase3): implications for NTPDase structural modeling. Biochemistry 44: 89989012.

Jones DT (1999) Protein secondary structure prediction based on position-specific scoring matrices. J Mol Biol 292: 195-202.

Kabsch W, Mannherz HG, Suck D, Pai EF, Holmes KC (1990) Atomic structure of the actin : DNase I complex. Nature 347: 37-44.

Kirley TL, Crawford PA, Smith TM (2006) The structure of the nucleoside triphosphate diphosphohydrolases (NTPDases) as revealed by mutagenic and computational modeling analyses. Purinergic Signalling 2: 379 389.

Komoszyński MA (1996) Comparative studies of animal and plant apyrases (ATP diphosphohydrolases EC 3.6.1.5) with application on immunological techniques and various ATPase inhibitors. Comp Biochem Physiol 113B: 581-591.

Komoszyński M, Wojtczak A (1996) Apyrase (ATP diphosphohydrolases, EC 3.6.1.5) function and relationship to ATPases. Biochim Biophys Acta 1310: 233-241.

Kraulis PJ (1991) MOLSCRIPT: a program to produce both detailed and schematic plots of protein structures. $J$ Appl Cryst 24: 946-950.

Kristensen O, Laurberg M, Liljas A, Kastrup JS, Gajhede M (2004) Structural characterization of the stringent response related exopolyphosphatase/guanosine pentaphosphate phosphohydrolase protein family. Biochemistry 43: 8894-8900.

Kukulski F, Komoszyński M (2003) Purification and characterization of NTPDase1 (Ecto-apyrase) and NTPDase2 (Ecto-ATPase) from porcine brain cortex synaptosomes. Eur J Biochem 270: 3447-3454.

Kukulski F, Levesque SA, Lavoie EG, Lecka J, Bigonnesse F, Knowles AF, Robson SC, Kirley TL, Sevigny J (2005) Comparative hydrolysis of P2 receptor agonists by NTPDases 1, 2, 3 and 8. Purinergic Signal 1: 193-204.

Kurowski MA, Bujnicki JM (2003) GeneSilico protein structure prediction meta-serve. Nucleic Acids Res 31: 33053307.

Laskowski RA, MacArthur M, Moss DS, Thornton JM (1993) PROCHECK: a program to check the stereochemical quality of protein structures. J Appl Cryst 26: 283-291. 
Lavoie EG, Kukulski F, Levesque SA, Lecka J, Sevigny J (2004) Cloning and characterization of mouse nucleoside triphosphate diphosphohydrolase-3. Biochemical Pharmacology 67: 1917-1926.

Mayer MP, Bukau B (2005) Hsp70 chaperones: cellular function and molecular mechanism. Cell Mol Life Sci 62: 670-684.

McGuffin LJ, Jones DT (2003) Improvement of the GenTHREADER method for genomic fold recognition. Bioinformatics 19: 874-881.

Merritt EA, Bacon DJ (1997) Raster3D: Photorealistic molecular graphics. Meth Enzymol 277: 505-524.

Notredame C, Higgins DG, Heringa J (2000) T-coffee: a novel method for fast and accurate multiple sequence alignment. J Mol Biol 302: 205-217.

O'Brien MC, Flaherty KM, McKay DB (1996) Lysine 71 of the chaperone protein Hsc70 is essential for ATP hydrolysis. J Biol Chem 271: 15874-15878.

Oses JP, Cardoso CM, Germano RA, Kirst IB, Rucker B, Furstenau CR, Wink MR, Bonan CD, Battastini AMO, Sarkis JJF (2004) Soluble NTPDase: An additional system of nucleotide hydrolysis in rat blood serum. Life Sciences 74: 3275-3284.

Phillips JC, Braun R, Wang W, Gumbart J, Tajkhorshid E, Villa E, Chipot Ch, Skeel RD, Kale L, Schulten K (2005) Scalable molecular dynamics with NAMD. J Comput Chem 26: 1781-1802.

Robson SC, Sévigny J, Zimmermann H (2006) The E-NTPDase family of ectonucleotidases: Structure function relationships and pathophysiological significance. $\mathrm{Pu}$ rinergic Signalling 2: 409-430.

Rychlewski L, Jaroszewski L, Li W, Godzik A (2000) Comparison of sequence profiles. Strategies for structural predictions using sequence information. Protein Sci 9: 232-241.

Schüler H (2001) ATPase activity and conformational changes in the regulation of actin. Biochim Biophysi Acta 1549: 137-147.

Schüler H, Korenbaum E, Schutt CE, Lindberg U, Karlsson R (1999) Mutational analysis of Ser14 and Asp157 in the nucleotide-binding site of $\beta$-actin. Eur J Biochem 265: $210-220$.
Shi J, Blundell TL, Mizuguchi K (2001) FUGUE: sequencestructure homology recognition using environmentspecific substitution tables and structure-dependent gap penalties. J Mol Biol 310: 243-257.

Sippl MJ (1993) Recognition of errors in three-dimensional structures of proteins. Proteins 17: 355-362.

Smith TM, Kirley TL (1999) Site-directed mutagenesis of a human brain ecto-apyrase: evidence that the E-type ATPases are related to the actin/heat shock 70/sugar kinase superfamily. Biochemistry 38: 321-328.

Smith TM, Lewis CSA, Kirley TL (1999) Mutagenesis of two conserved tryptophane residues of the E-type ATPases. Inactivation and conversion of an Ecto-Apyrase to an Ecto-NTPase. Biochemistry 38: 5849-5857.

Sondermann H, Scheufler C, Schneider C, Hohfeld J, Hartl FU, Moarefi I (2001) Structure of a Bag/Hsc70 complex: convergent functional evolution of Hsp70 nucleotide exchange factors. Science 291: 1553-1557.

Sousa MC, MacKay DB (1998) The hydroxyl of threonine 13 of the bovine $70-\mathrm{kDa}$ heat shock cognate protein is essential for transducing the ATP-induced conformational change. Biochemistry 37: 15392-15399.

Sriram M, Osipiuk J, Freeman BC, Morimoto RI, Joachimiak A (1997) Human Hsp70 molecular chaperone binds two calcium ions within the ATPase domain. Structure 5: 403-414.

Vorobiev S, Strokopytov B, Drubin DG, Frieden C, Ono J, Condeelis J, Rubenstein PA, Almo SC (2003) The structure of nonvertebrate actin: implications for the ATP hydrolytic mechanism. Proc Natl Acad Sci USA 100: 5760-5765.

Wilbanks SM, DeLuca-Flaherty C, McKay DB (1994) Structural basis of the 70-kilodalton heat shock cognate protein ATP hydrolytic activity. I. Kinetic analyses of active site mutants. J Biol Chem 269: 12893-12898.

Yang W, Hendrickson WA, Crouch RJ, Satow Y (1990) Structure of ribonuclease $\mathrm{H}$ phased at $2 \AA$ resolution by MAD analysis of the selenomethionyl protein. Science 249: 1398-1405.

Yang F, Hicks-Berger CA, Smith TM, Kirley TL (2001) Sitedirected mutagenesis of human nucleoside triphosphate diphosphohydrolase3: The importance of residues in the apyrase conserved regions. Biochemistry 40: 3943-3950. 